\title{
Big data meets mechanism
}

\author{
Inflammatory disease research is burgeoning. Large data sets are being generated to characterize the human \\ immune response, while detailed mechanistic studies are defining the role of specific cell types and sensors in \\ inflammatory disease. Future efforts are needed to integrate these approaches and guide precision medicine.
}

mmune homeostasis requires calibrated responses aimed at defending the host, walling it off from infection and promoting repair from injury. However, this process can go awry, resulting in chronic inflammation. The specially commissioned articles in this Focus issue on inflammatory disease aim to highlight emerging research into this process and its complexity. This complexity exists at numerous levels, from the interplay between environmental factors, immune cell subsets, and cellular sensors and the mediators they produce. It is clear that chronic inflammation is the result of many moving parts, and that many layers and types of information are needed to understand it.

Two such moving parts are commensal microbes and dietary components. Advances in next-generation sequencing have enabled profiling of the microbiota in humans and how dysbiosis is associated with disease. The upstream causes of this dysbiosis, including environmental factors, perturbations induced by antibiotics and infection, as well as the dominant influence of early life exposure to microbes, are only now being teased apart. Metagenomics and metabolomics are enabling analyses of the gene products and metabolites produced by these microbes, with a detailed picture emerging regarding the abundance and function of microbes. Despite being produced in the gut, these microbial-derived metabolites act systemically, as highlighted in a News Feature (p 674). Moreover, Marc Veldhoen and Cristina Ferreira explain how dietary metabolites can alter immune cell development and function either directly or indirectly by altering the microbiota (p 709).

Most studies of the microbiota have focused on bacteria, but additional work is needed to profile all of the microbes present, and to determine how bacteria, fungi, parasites, viruses and bacteriophages interact with each other and the host to influence immune status. The success of fecal microbial transplantation for the treatment of Clostridium difficile infections points to the importance of human studies to define what constitutes a healthy microbiota, which species confer protection upon microbial transfer, and how to rationally design an effective probiotic or prebiotic. Moving forward, an iterative process of using data in human cohorts to inform mechanistic work and hypothesis testing in germ-free mice and other preclinical models may provide a clear path through the complexity.

Although the gut microbiota can affect host physiology systemically, microbes are present at all barrier surfaces, including the skin, lungs, genitourinary tract and the oral cavity. Whether these have a tissue-specific role in modulating immune responses is currently being investigated. Two particular cell types enriched at barrier surfaces-innate lymphoid cells (ILCs) and tissue-resident memory T cells-are discussed in this issue by Gregory Sonnenberg and David Artis (p 698) and Thomas S. Kupper and Chang Ook Park (p 688), respectively. Tissue-specific immune cells present at distinct anatomical sites exhibit functional and phenotypic heterogeneity, and they are conditioned by cues in the tissue microenvironment (e.g., hypoxia, microbes and dietary components), which imprint homing properties and functional specialization. Studies using mass cytometry (or CyTOF), and epigenetics, transcriptome and metabolic analyses are helping delineate how the microenvironment modulates immune cell function, how these cells adapt, and the degree of plasticity between cell types.

Complexity is also seen at the level of innate immune sensors. As discussed by Jenny Ting and colleagues (p 677), the vast array of different inflammasome complexes that assemble in response to distinct pathogens and host factors illustrates the diversity and specialization of this system. Moreover, new research is highlighting inflammasome-independent functions of scaffold proteins that sense or bind activating stimuli, suggesting that their roles extend beyond the production of inflammatory mediators. Additionally, small-molecule inhibitors that only target specific inflammasome components may attenuate inflammatory disease without attendant impairments in host defense to infection.

Therapeutically, inflammatory mediators secreted in response to activation of these sensors are quite effective drug targets, with sales exceeding $\$ 25$ billion per year for tumor necrosis factor (TNF) inhibitors. In addition to biologics targeting interleukin (IL)-12/23p40, IL-23p19 and IL-17, as discussed by Daniel Cua and colleagues ( $p$ 719), the notion of targeting a single cytokine in several phenotypically distinct diseases points to potentially shared mechanisms underlying these diseases. This suggests that treatment decisions should be guided less by clustering diseases on the basis of related symptoms and more by a mechanistic understanding of disease etiology. Accordingly, genome-wide association studies have also revealed a common immune-mediated basis for seemingly disparate autoimmune diseases, as discussed by Judy Cho and Marc Feldmann (p 730).

Our understanding of inflammatory disease etiology will be boosted further by studies using multiple omics platforms. By embracing big data and integrating host genetics with longitudinal proteomics, metabolomics, immune cell phenotyping, microbiome and clinical data, the extent to which host genetics modulate immune responses, and whether any particular factors are associated with disease may become apparent. Longitudinal data sets will also be helpful resources to define what constitutes a healthy human immune system, the degree of variability between individuals, and how host-environment interactions shape immune responses, disease susceptibility and response to therapy. If begun shortly after birth, these data sets might even allow us to track the development of the immune system. Future insights into inflammatory disease can thus be accelerated by unbiased approaches aimed at assessing the immune response, while in parallel investigating mechanistically how these factors modulate immune homeostasis in preclinical models. In the future, disease heterogeneity may be parsed and response to therapy predicted, thereby enabling precision medicine.

In closing, we thank Janssen Pharmaceutical Companies of Johnson \& Johnson for the financial support to produce this special issue of the journal. As always, Nature Medicine takes full editorial responsibility for the content of these pages. 